\title{
Prevalence of Prescription Opioids for Nonoperative Treatment of Rotator Cuff Disease Is High
}

\author{
Jacob Gorbaty, M.D., Susan M. Odum, Ph.D., Meghan K. Wally, M.S.P.H., \\ Rachel B. Seymour, Ph.D., Nady Hamid, M.D., Joseph R. Hsu, M.D., and the PRIMUM \\ Group
}

\begin{abstract}
Purpose: To quantify the prevalence of opioid and benzodiazepine prescriptions for patients with rotator cuff disease across a large health care system and to describe evidence-based risk factors for opioid use within this population. Methods: We conducted a retrospective cohort study at a major health care system of all patients with qualifying diagnostic codes. Emergency department, urgent care, and outpatient encounters between January and December 2016 for an acute rotator cuff tear, listed as the primary diagnosis, were included. Encounters with prescriptions for opioids or benzodiazepines were identified using the Prescription Reporting With Immediate Medication Utilization Mapping (PRIMUM) system. Descriptive statistics and the rate of controlled-substance prescribing were calculated for the population as a whole and among subgroups. Results: We identified 9,376 encounters meeting the inclusion criteria. Of these encounters, $1,559(16.6 \%)$ resulted in 1 or more prescriptions for an opioid or benzodiazepine that were issued during the visit. A total of 2,007 opioid and/or benzodiazepine prescriptions were issued for the 1,559 encounters (rate of 1.29 prescriptions per prescribing encounter). This represented 5,310 patients, of whom 1,096 (20.6\%) received a prescription for an opioid or benzodiazepine during at least 1 of their encounters. Of patients who received a prescription, $20.9 \%$ had at least 1 risk factor for prescription misuse; $3.6 \%$ of patients had more than 1 risk factor. There were no demographic differences between patients with risk factors and patients without them. Conclusions: The prescribing of opioids for the treatment of pain in patients with rotator cuff disease remains high across multiple locations and specialties within a large health care system. Using alternative pain management pathways as primary prevention for opioid misuse and abuse in high opioid-prescribing locations - and especially for patients identified as having a high risk of opioid misuse-is an important practice to continue in our shift away from opioid use as a health care system. Level of Evidence: Level IV, case series.
\end{abstract}

From the Department Orthopaedic Surgery, Atrium Health Musculoskeletal Institute, Charlotte, North Carolina, U.S.A. (J.G., S.M.O., M.K.W., R.B.S., N.H., J.R.H., M.J.B., D.L., T.R., Z.Y.); OrthoCarolina Shoulder and Elbow Center, Charlotte, North Carolina, U.S.A. (N.H.); NC Poison Control, Atrium Health, Charlotte, North Carolina, U.S.A. (M.B.); Department of Emergency Medicine, Carolinas Trauma Network Research Center of Excellence, Atrium Health, Charlotte, North Carolina, U.S.A. (M.G., C.G., M.R.); Patient Safety, Atrium Health, Charlotte, North Carolina, U.S.A. (S.J.); Department of Internal Medicine, Atrium Health, Charlotte, North Carolina, U.S.A. (A.S.); US Acute Care Solutions, Atrium Health, Pineville, North Carolina, U.S.A. (B.W.); and Adult Psychiatry, Atrium Health, Charlotte, North Carolina, U.S.A. (S.W.).

The PRIMUM (Prescription Reporting With Immediate Medication Utilization Mapping) Group comprises Michael Beuhler, M.D., Michael J. Bosse, M.D., Michael Gibbs, M.D., Christopher Griggs, M.D., Steven Jarrett, Pharm.D., Daniel Leas, M.D., Tamar Roomian, M.S., M.P.H., Michael Runyon, M.D., Animita Saha, M.D., Bradley Watling, M.D., Stephen Wyatt, D.O., and Ziqing Yu, M.S.

The authors report the following potential conflicts of interest or sources of funding: The Centers for Disease Control and Prevention (CDC) provided grant funding in support of this work. S.M.O. is a paid consultant for American Academy of Orthopaedic Surgeons, outside the submitted work.
M.K.W. receives grant support from the CDC. R.B.S. receives grant support from the CDC. J.R.H. receives grant support from the CDC. In addition, J.R.H. is on the speakers bureau of Smith $\theta$ Nephew and is a consultant for Globus Medical, outside the submitted work. M.B. receives grant support from the $C D C$. C.G. receives grant support from the $C D C$. In addition, C.G. receives grant support from Amerisource Bergen/Boston University, outside the submitted work. S.J. receives grant support from the CDC. D.L. receives grant support from the CDC. T.R. receives grant support from the CDC. M.R. receives grant support from the CDC. A.S. receives grant support from the CDC. B.W. receives grant support from the $C D C$. S.W. receives grant support from the $C D C$. Z.Y. receives grant support from the CDC. Full ICMJE author disclosure forms are available for this article online, as supplementary material.

Received April 17, 2020; accepted September 30, 2020.

Address correspondence to Rachel B. Seymour, Ph.D., 1320 Scott Ave, Charlotte, NC 28204,U.S.A.E-mail: rachel.seymour@atriumhealth.org

(C) 2021 THE AUTHORS. Published by Elsevier Inc. on behalf of the Arthroscopy Association of North America. This is an open access article under the CC BY-NC-ND license (http://creativecommons.org/licenses/by-nc-nd/4.0/). 2666-061X/20478

https://doi.org/10.1016/j.asmr.2020.09.028 
$\mathbf{O}^{2}$ pioid prescriptions and use continue to be a topic of intense scrutiny. Unfortunately, there is still little guidance for the treatment of mild to moderate musculoskeletal pain, such as rotator cuff disease. Prescribers who first come into contact with patients with rotator cuff disease include primary care physicians, emergency and urgent care clinicians, advanced practice providers, and surgical specialists such as orthopaedic surgeons. Most of the prescription pain medications prescribed by orthopaedic surgeons are schedule II and III medications, both of which have high potential for abuse and dependence. ${ }^{1}$

Evidence-based guidelines do not support long-term improvements in pain or function in patients with musculoskeletal pain when treated with opioids. The American Academy of Orthopaedic Surgeons appropriate-use criteria for optimizing management of full-thickness rotator cuff tears recommends "NSAIDs [nonsteroidal anti-inflammatory drugs], Ultram (Janssen, Titusville, NJ), light narcotics for short time periods, [a] steroid dose pack," and corticosteroid injections for nonoperative medical management. ${ }^{2}$ The recommendation is intentionally broad to "allow clinical judgment, expertise and physician training/preference to play a role in optimal treatment selection." ${ }^{2}$ This recommendation does not include partial-thickness tears or other conditions such as impingement that may be included within the term "rotator cuff disease," although it may be inferred that increased narcotics would not be recommended for less severe forms of the condition.

Across orthopaedics, multiple studies have shown that preoperative opioid use may be associated with worse outcomes, as well as increased postoperative opioid use and other pain management requirements. ${ }^{3-5}$ Recent research has shown that patients undergoing rotator cuff repair who were prescribed preoperative opioids showed less improvement in external rotation as well as lower preoperative and postoperative American Shoulder and Elbow Surgeons, Constant, and Simple Shoulder Test scores. ${ }^{6,7}$

Some attempts have been made to change the course for these patients by targeting preoperative use. Syed et al., ${ }^{8}$ in a randomized clinical trial, showed that preoperative education led to significantly less narcotic use in the 3-month postoperative period; in addition, patients with preoperative narcotic use were 6.8 times more likely to discontinue narcotics by the end of follow-up.

Other efforts have attempted to better define risk factors and describe opioid prescribing and use within the musculoskeletal population to direct future interventions and increase their efficacy. Alamanda et al. ${ }^{9}$ recently attempted to quantify the prevalence of opioid and benzodiazepine prescribing for the diagnosis of osteoarthritis from all prescribers over a l-year period across a large health care system. Examining rotator cuff disease, Westermann et al. ${ }^{10}$ have reported on the cumulative incidence of opioid prescriptions specifically written for patients after rotator cuff repair.

Unfortunately, there is a lack of universal prescribing recommendations for common musculoskeletal conditions, and prescribing habits tend to vary widely among orthopaedic surgeons, with many still prescribing more opioids for postoperative rotator cuff repair than are necessary. ${ }^{11,12}$ There is still a need for a cohesive understanding of the pattern of opioid prescribing surrounding rotator cuff disease. Additionally, there are known risk factors for opioid misuse and abuse, but the prevalence of these risk factors in this population and the association with the receipt of an opioid prescription are relatively unknown.

The purposes of this study were to quantify the prevalence of opioid and benzodiazepine prescriptions for patients with rotator cuff disease across a large health care system and to describe evidence-based risk factors for opioid use within this population. We hypothesized that a portion of patients with rotator cuff disease would receive opioids or benzodiazepines and that some of these patients would be at higher risk of opioid use disorder.

\section{Methods}

A retrospective cohort study at a major health care system was conducted to assess the prescribing patterns of opioids and benzodiazepines for patients with a primary diagnosis of rotator cuff disease. Study approval was obtained from the Prescription Reporting With Immediate Medication Utilization Mapping (PRIMUM) internal review board. Emergency department, urgent care, and outpatient encounters between January and December 2016 for patients with acute rotator cuff tears, defined as those with 3 specific International Classification of Diseases, Tenth Revision codes listed as the primary diagnosis (M75.1, M75.4, and M75.8), were included in the study. Each visit was pulled as a separate encounter. Encounters for outpatient surgery and inpatient stays associated with a primary diagnosis of rotator cuff tear were excluded.

Encounters with prescriptions for opioids or benzodiazepines were identified using the PRIMUM system. The PRIMUM system is a clinical decision support system integrated in the electronic medical record (EMR) that was developed to address patient safety by providing critical, objective patient risk information at the point of care. ${ }^{13}$ The PRIMUM system presents an alert to prescribers when patients meet 1 or more of the following criteria ("triggers"): early refill $(>50 \%$ of existing opioid or benzodiazepine prescription remaining); 2 or more onsite administrations of opioids or benzodiazepines within the previous 30 days; 3 or more 
opioid or benzodiazepine prescriptions in the previous 30 days; history of overdose; and positive blood alcohol, marijuana, or cocaine toxicology screening results. The triggers used by the PRIMUM database and its associated EMR alert system are known evidence-based risk factors for opioid misuse or abuse and/or diversion of prescription narcotics. ${ }^{14-21}$ The characteristics of the 5 known risk factors chosen to be triggers in this system include objective factors that have consistent and accurate documentation in the EMR in a specific location that can be searched for in an automated fashion. ${ }^{13}$

Encounter data for all patients with qualifying diagnostic codes and data from the PRIMUM system were merged to include all patients with rotator cuff disease, including those who received prescription opioids or benzodiazepines and those who did not. Morphine milligram equivalents (MMEs) were calculated for each prescription. $^{22}$ Descriptive statistics were used to characterize these patient populations regarding demographic characteristics, type of treatment facility, and type of prescriber. The rate of controlled-substance prescribing was calculated for the population as a whole and among subgroups, such as age groups, locations, and patient risk factors.

\section{Statistical Analysis}

Descriptive statistics were used to describe the demographic characteristics and risk factors of this patient population, as well as the distribution of opioid and benzodiazepine prescribing and doses. The KruskalWallis, Mann-Whitney $U$, and $\chi^{2}$ tests were used to determine associations between opioid prescribing patterns, patient risk factors, and average daily MMEs by patient, prescriber, and encounter characteristics. All statistical analyses were conducted using SAS software (version 9.4 [2012]; SAS Institute, Cary, NC).

\section{Results}

After the exclusion of 1,336 encounters because of outpatient surgery and 1,672 encounters because of inpatient stays, a total of 9,376 encounters met our inclusion criteria. Of these encounters, 1,559 (16.6\%) resulted in 1 or more prescriptions for an opioid or benzodiazepine that was issued during the visit. A total of 2,007 opioid and/or benzodiazepine prescriptions were issued for the 1,559 encounters (rate of 1.29 prescriptions per prescribing encounter). This represented 5,310 patients, of whom 1,096 (20.6\%) received a prescription for an opioid or benzodiazepine during at least 1 of their encounters. Of the patients, $30.1 \%$ had more than 1 encounter during the study period.

The characteristics of the patients with rotator cuff disease are presented in Table $1(\mathrm{~N}=5,310)$. A smaller proportion of patients aged 68 years or older received an opioid $(16.9 \%)$ as compared with patients aged 49 to
58 years $(25.4 \%, P=.0001)$. Regarding race, $22.7 \%$ of nonwhite patients received an opioid versus $19.8 \%$ of white patients $(P=.0204)$. There was no significant difference by sex. Patients with only 1 encounter during the study period were less likely to receive an opioid $(15.3 \%)$ than those with more than 1 encounter $(33 \%)$ $(P<.0001)$.

The characteristics of the patients with rotator cuff disease who received a prescription $(n=1,096)$ are presented in Table 2. About one-fifth of patients who received a prescription $(20.9 \%)$ had at least 1 risk factor for prescription misuse (Table 2); 3.6\% of patients had more than 1 risk factor. The most common trigger among patients of all ages was an early refill (14\%), followed by 3 or more prescriptions in the last 30 days $(6 \%)$ and positive toxicology (4\%). Onsite administration and a history of overdose were rare in this population $(0.6 \%$ and $0.4 \%$, respectively). There were no demographic differences between patients who had a risk factor and those without risk factors.

The characteristics of all prescribing encounters are presented in Table 3. Approximately 15\% of prescribing encounters were in the urgent care or emergent care setting, whereas the rest were in the outpatient clinic setting. Most prescribing encounters $(53 \%)$ were with prescribers in medical or surgical specialties, with approximately $25 \%$ of the encounters conducted in the primary care setting (i.e., family medicine or internal medicine). Of note, $10 \%$ of patient encounters originated from pain management clinics. A significant portion of all prescribing encounters $(33 \%)$ were with advanced care practitioners (nurse practitioners and physician assistants). Patients were more likely to receive an opioid prescription if presenting to emergency department or urgent care facilities $(52.6 \%$ of encounters) compared with outpatient facilities $(14.1 \%)(P<.0001)$.

Analysis of the medications prescribed revealed that prescriptions were written for a median of 30 daily MMEs and 16 days. Hydrocodone-acetaminophen was the most commonly prescribed medication $(37 \%)$, with a median daily MME of 30 (Table 4). Of the opioid prescriptions, $35 \%$ were for "potent opioids" (morphine milliequivalent conversion factor $>1$ ). The most common potent opioid prescribed was oxycodone-acetaminophen (24\%; median, $60 \mathrm{MME} / \mathrm{d}$ ).

\section{Discussion}

Despite the mounting evidence against opioid prescribing for minor musculoskeletal conditions, over one-fifth of all patients with a primary diagnosis of rotator cuff disease received a narcotic or opioid prescription during an encounter with a prescriber over the course of 12 months. Of those who received an opioid, nearly 1 in 5 had at least 1 risk factor for opioid misuse, and of the opioids prescribed, over one-third were 
Table 1. Patient Demographic Characteristics

\begin{tabular}{|c|c|c|c|c|c|c|c|}
\hline & \multicolumn{2}{|c|}{ All $(\mathrm{N}=5,310)$} & \multicolumn{2}{|c|}{ Opioid Not Prescribed $(n=4,214)$} & \multicolumn{2}{|c|}{ Opioid Prescribed $(n=1,096)$} & $P$ Value \\
\hline Age group & & & & & & & .0001 \\
\hline $49-58 \mathrm{yr}$ & 1,447 & 27.3 & 1,080 & 74.6 & 367 & 25.4 & \\
\hline $59-67 \mathrm{yr}$ & 1,062 & 20.0 & 846 & 79.7 & 216 & 20.3 & \\
\hline$\geq 68$ yr & 1,445 & 27.2 & 1,201 & 83.1 & 244 & 16.9 & \\
\hline Sex & & & & & & & .44 \\
\hline Race & & & & & & & .0204 \\
\hline Nonwhite & 1,517 & 28.6 & 1,173 & 77.3 & 344 & 22.7 & \\
\hline White & 3,793 & 71.4 & 3,041 & 80.2 & 752 & 19.8 & \\
\hline No. of encounters & & & & & & & .0001 \\
\hline$\geq 2$ & 1,597 & 30.1 & 1,070 & 67.0 & 527 & 33.0 & \\
\hline 1 & 3,713 & 69.9 & 3,144 & 84.7 & 569 & 15.3 & \\
\hline
\end{tabular}

NOTE. There was a significant difference $(P=.0001)$ among age groups between patients who received an opioid prescription and those who did not. Nonwhite patients were more likely to receive opioids $(P=.0204)$ compared with white patients. Patients with multiple encounters were more likely to receive an opioid prescription compared with patients with a single encounter $(P=.0001)$.

potent in nature. The health care system studied for this cohort consists of 6 freestanding emergency departments, 28 urgent care locations, and over 500 physician practices with over 3,500 physicians and other prescribers using a common EMR. These results thus likely capture a high percentage of all prescriptions for this patient population in this geographic area and are likely representative of national trends.

It has previously been shown that preoperative opioid use has adverse effects on postoperative effects after several orthopaedic procedures including knee arthroplasty, spine surgery, and total shoulder arthroplasty. 3 23-25 These adverse effects include increased hospital stays and reoperation rates, as well as worse outcome scores. In a recent series examining outcomes after anatomic total shoulder arthroplasty, Thompson et al. ${ }^{26}$ showed that, even when controlling for confounding variables, such as age, body mass index, sex,

Table 2. Patient Risk Factors Among Patients Prescribed Opioids

\begin{tabular}{lc}
\hline & $\mathrm{n}(\%)$ \\
\hline Risk factor & $148(14)$ \\
Early refill & $7(0.6)$ \\
Onsite administration of opioid or benzodiazepine & $70(6)$ \\
$\geq 3$ prescriptions & $48(4)$ \\
Positive toxicology & $4(0.4)$ \\
History of overdose & \\
No. of risk factors & $867(79.1)$ \\
0 & $190(17.3)$ \\
1 & $37(3.4)$ \\
2 & $2(0.2)$ \\
3 &
\end{tabular}

NOTE. Among patients who were prescribed an opioid, $23.9 \%$ had at least 1 risk factor. The most common risk factor was an early refill (14\%), followed by 3 or more prescriptions $(6 \%)$ and positive toxicology $(4 \%)$. chronic pain syndromes, psychiatric conditions, and comorbidities, preoperative opioid use (within 3 months of surgery) was an independent negative prognostic indicator of outcomes including American Shoulder and Elbow Surgeons scores, range of motion, strength, and visual analog scale scores when compared with a non-narcotic group.

Attempts have been made to predict which preoperative factors infer a higher likelihood of postoperative opioid use. Westermann et al. ${ }^{10}$ published research using a large single-insurer database examining the cumulative incidence of opioid prescriptions given to patients having undergone rotator cuff repair (using Current Procedural Terminology code 29827). They found that patients who filled an opioid prescription 1 to 3 months before surgery were 7.45 times more likely to fill a prescription 3 months after surgery compared with patients who were not prescribed an opioid before surgery. Other risk factors for prolonged opioid use were diagnoses of depression, anxiety, low-back pain, and myalgia. ${ }^{10}$ It is interesting to note that their data showed a preoperative opioid use rate of $43 \%$, over twice the rate reported in the PRIMUM database. Data from the PRIMUM database capture all patients who received opioids at any time and do not further separate patients based on future procedures or the lack thereof. Not all patients with rotator cuff pathology in the PRIMUM database who received opioids went on to surgery. Considering the data from Westermann et al., the findings may indicate that patients who are surgical candidates are more likely to receive preoperative opioids. Additionally, encounters that occurred at outpatient surgical centers were excluded from the PRIMUM database. It follows that patients should be counseled about the risk of increased postoperative opioid demand when taking narcotic medications before surgery. 
Table 3. Prescribing Encounter Characteristics

\begin{tabular}{lr}
\hline & $\mathrm{n}(\%)$ \\
\hline Location & $237(15)$ \\
ED or urgent care & $1,322(85)$ \\
Outpatient & \\
Specialty & $180(12)$ \\
Emergency & $156(10)$ \\
Pain & $385(25)$ \\
Primary care & $167(11)$ \\
Medical & $655(42)$ \\
Surgical & $15(1)$ \\
Unknown & \\
Prescriber type & $390(25)$ \\
Advanced practice provider & $1,039(67)$ \\
Physician & $130(8)$ \\
Other &
\end{tabular}

NOTE. Characteristics are presented for the encounters in which opioids were prescribed. Most encounters $(85 \%)$ occurred in an outpatient setting; $42 \%$ of encounters occurred in surgical specialties compared with $25 \%$ occurring in primary care specialties; and $67 \%$ of prescribing encounters were conducted by physicians.

ED, emergency department.

Looking for risk factors for opiate use beyond 90 days, Basilico et al. ${ }^{27}$ examined 17,961 opiate-naive patients treated for musculoskeletal injury from an institutional database. When adjusted for discharge MMEs, only opioid quantity was predictive of prolonged use. However, more potent opioids were associated with higher discharge MMEs. Analysis of the PRIMUM database shows that potent opioids still make up a large proportion of the opioids prescribed. This is an area in which improvement can easily be made through education and information dissemination. The PRIMUM database furthermore allows investigators to track these efforts for opioid reduction across the health care system.

Alternative treatment modalities that can be implemented to help manage symptoms include the use of NSAIDs, acetaminophen, cryotherapy, transcutaneous electrical nerve stimulation, and cognitive behavioral therapy. ${ }^{28-30}$ In a systematic review and meta-analysis on the efficacy of oral NSAIDs for rotator cuff tendinopathy, Boudreault et al. ${ }^{31}$ found that oral NSAIDs provide significant pain relief compared with placebo. They also found that NSAIDs in the short term are as effective as corticosteroid injections. Of the high-quality studies compiled, most were underpowered to detect differences in adverse events, and they did not compare NSAIDs directly with narcotic medications, leaving room for future research.

The American Academy of Orthopaedic Surgeons has published evidence-based guidelines on the nonsurgical management of osteoarthritis in the knee, with recommendations for low-impact aerobic and strengthening exercises and recommendations against opioids for symptomatic treatment. ${ }^{32}$ There are no clear guidelines for or against the use of opioids for treatment of rotator cuff pathology. Despite this, clinicians should be discouraged from prescribing opioid pain medication for rotator cuff pathology regardless of whether the patient will be an operative candidate.

\section{Limitations}

Regarding study limitations, first, this is a retrospective study in nature and is limited to prescriptions provided for outpatient encounters within the health care system. Second, owing to the nature of the database, the data are dependent on the input and detail provided by many individuals across the system. Granularity is therefore limited to the accuracy of the group as a whole, and it is difficult to define more patient-specific factors such as chronicity of the condition or comorbidities. Finally, data collection was restricted to the prescription of opioids and benzodiazepines and therefore does not capture whether the prescriptions were filled and how they were used. Additional prescriptions for other medications, such as non-opioid analgesics, prescribed for an encounter were not recorded owing to the design of the automated data capture and because these medications are also available over the counter.

Table 4. Characteristics of Prescriptions

\begin{tabular}{|c|c|c|c|c|}
\hline & $\mathrm{n}(\%)$ or $\mathrm{n}$ & Median (MME) & IQR (MME) & Range (MME) \\
\hline Daily MMEs & 1,877 & 30 & $20-60$ & $3.8-360$ \\
\hline Duration of prescription & 2,007 & $16 \mathrm{~d}$ & $10-30 \mathrm{~d}$ & $1.0-240 \mathrm{~d}$ \\
\hline \multicolumn{5}{|l|}{ Medication } \\
\hline Fentanyl & $40(2)$ & 120 & $60-270$ & $28.8-270$ \\
\hline Hydrocodone-acetaminophen & $739(37)$ & 30 & $20-40$ & $5-60$ \\
\hline Oxycodone & $173(9)$ & 90 & $45-135$ & $7.5-360$ \\
\hline Other opioid & $147(7)$ & 60 & $30-90$ & $3.8-320$ \\
\hline
\end{tabular}

NOTE. The most common opioid prescribed was hydrocodone-acetaminophen (37\%), followed by oxycodone-acetaminophen $(24 \%)$. The median duration of prescriptions was 16 days.

IQR, interquartile range; MME, morphine milligram equivalent; NA, not applicable. 


\section{Conclusions}

The prescribing of opioids for the treatment of pain in patients with rotator cuff disease remains high across multiple locations and specialties within a large health care system. Using alternative pain management pathways as primary prevention for opioid misuse and abuse in high opioid-prescribing locations-and especially for patients identified as having a high risk of opioid misuse-is an important practice to continue in our shift away from opioid use as a health care system.

\section{References}

1. Morris BJ, Mir HR. The opioid epidemic: Impact on orthopaedic surgery. J Am Acad Orthop Surg 2015;23:267-271.

2. Pappou I, Schmidt C, Jarrett C, Steen B, Frankle M. AAOS appropriate use criteria: Optimizing the management of full-thickness rotator cuff tears. J Am Acad Orthop Surg 2013;21:772-775.

3. Morris BJ, Sciascia AD, Jacobs CA, Edwards TB. Preoperative opioid use associated with worse outcomes after anatomic shoulder arthroplasty. J Shoulder Elbow Surg 2016;25:619-623.

4. Franklin PD, Karbassi JA, Li W, Yang W, Ayers DC. Reduction in narcotic use after primary total knee arthroplasty and association with patient pain relief and satisfaction. J Arthroplasty 2010;25:12-16 (suppl).

5. Pivec R, Issa K, Naziri Q, Kapadia BH, Bonutti PM, Mont MA. Opioid use prior to total hip arthroplasty leads to worse clinical outcomes. Int Orthop 2014;38:1 159-1165.

6. Williams BT, Redlich NJ, Mickschl DJ, Grindel SI. Influence of preoperative opioid use on postoperative outcomes and opioid use after arthroscopic rotator cuff repair. J Shoulder Elbow Surg 2019;28:453-460.

7. Sabesan VJ, Petersen-Fitts GR, Sweet MC, Katz DL, Lima DJL, Whaley JD. The impact of preoperative opioid use on outcomes after arthroscopic rotator cuff repair. JSES Open Access 2018;2:155-158.

8. Syed UAM, Aleem AW, Wowkanech C, et al. Neer Award 2018: The effect of preoperative education on opioid consumption in patients undergoing arthroscopic rotator cuff repair: A prospective, randomized clinical trial. J Shoulder Elbow Surg 2018;27:962-967.

9. Alamanda VK, Wally MK, Seymour RB, Springer BD, Hsu JR, Prescription Reporting With Immediate Medication Utilization Mapping Group. Prevalence of opioid and benzodiazepine prescriptions for osteoarthritis. Arthritis Care Res (Hoboken) 2020;72:1081-1086.

10. Westermann RW, Anthony CA, Bedard N, et al. Opioid consumption after rotator cuff repair. Arthroscopy 2017;33: 1467-1472.

11. Bedard NA, Sierra RJ, Mabry T. Opioids after orthopaedic surgery: There is a need for universal prescribing recommendations: Commentary on an article by Matthew J. Sabatino, MD, MS, et al.: “Excess Opioid Medication and Variation in Prescribing Patterns Following Common Orthopaedic Procedures." J Bone Joint Surg Am 2018;100:el 7.

12. Sabatino MJ, Kunkel ST, Ramkumar DB, Keeney BJ, Jevsevar DS. Excess opioid medication and variation in prescribing patterns following common orthopaedic procedures. J Bone Joint Surg Am 2018;100:180-188.

13. Seymour RB, Leas D, Wally MK, Hsu JR, PRIMUM Group. Prescription reporting with immediate medication utilization mapping (PRIMUM): Development of an alert to improve narcotic prescribing. BMC Med Inform Decis Mak 2016;16:111.

14. Yang Z, Wilsey B, Bohm M, et al. Defining risk of prescription opioid overdose: Pharmacy shopping and overlapping prescriptions among long-term opioid users in Medicaid. J Pain 2015;16:445-453.

15. Webster LR, Webster RM. Predicting aberrant behaviors in opioid-treated patients: Preliminary validation of the Opioid Risk Tool. Pain Med 2005;6:432-442.

16. Grover CA, Elder JW, Close RJ, Curry SM. How frequently are "classic" drug-seeking behaviors used by drug-seeking patients in the emergency department? West J Emerg Med 2012;13:416-421.

17. Paulozzi LJ, Kilbourne EM, Shah NG, et al. A history of being prescribed controlled substances and risk of drug overdose death. Pain Med 2012;13:87-95.

18. Zedler B, Xie L, Wang L, et al. Risk factors for serious prescription opioid-related toxicity or overdose among Veterans Health Administration patients. Pain Med 2014;15:1911-1929.

19. Jones CM, Paulozzi LJ, Mack KA. Sources of prescription opioid pain relievers by frequency of past-year nonmedical use United States, 2008-2011. JAMA Intern Med 2014;174:802-803.

20. Weiner SG, Griggs CA, Langlois BK, et al. Characteristics of emergency department "doctor shoppers." J Emerg Med 2015;48:424-431el.

21. Hall AJ, Logan JE, Toblin RL, et al. Patterns of abuse among unintentional pharmaceutical overdose fatalities. JAMA 2008;300:2613-2620.

22. Centers for Medicare \& Medicaid Services. Opioid morphine equivalent conversion factors 2016, https://www.cms.gov/ Medicare/Prescription-Drug-Coverage/PrescriptionDrug CovContra/Downloads/Opioid-Morphine-EQ-ConversionFactors-Aug-2017.pdf. Accessed November 21, 2019.

23. Lee D, Armaghani S, Archer KR, et al. Preoperative opioid use as a predictor of adverse postoperative self-reported outcomes in patients undergoing spine surgery. J Bone Joint Surg Am 2014;96:e89.

24. Cheah JW, Sing DC, McLaughlin D, Feeley BT, Ma CB, Zhang AL. The perioperative effects of chronic preoperative opioid use on shoulder arthroplasty outcomes. J Shoulder Elbow Surg 2017;26:1908-1914.

25. Ben-Ari A, Chansky H, Rozet I. Preoperative opioid use is associated with early revision after total knee arthroplasty. J Bone Joint Surg Am 2017;99:1-9.

26. Thompson KM, Hallock JD, Smith RA, Brolin TJ, Azar FM, Throckmorton TW. Preoperative narcotic use and inferior outcomes after anatomic total shoulder arthroplasty: A clinical and radiographic analysis. J Am Acad Orthop Surg 2019;27:177-182.

27. Basilico M, Bhashyam AR, Harris MB, Heng M. Prescription opioid type and the likelihood of prolonged opioid use after orthopaedic surgery. J Am Acad Orthop Surg 2019;27:e423-e429. 
28. Uquillas CA, Capogna BM, Rossy WH, Mahure SA, Rokito AS. Postoperative pain control after arthroscopic rotator cuff repair. J Shoulder Elbow Surg 2016;25:1204-1213.

29. Mahure SA, Rokito AS, Kwon YW. Transcutaneous electrical nerve stimulation for postoperative pain relief after arthroscopic rotator cuff repair: A prospective double-blinded randomized trial. J Shoulder Elbow Surg 2017;26:1508-1513.

30. Oh JH, Seo HJ, Lee Y-H, Choi H-Y, Joung HY, Kim SH. Do Selective COX-2 inhibitors affect pain control and healing after arthroscopic rotator cuff repair? A preliminary study. Am J Sports Med 2018;46:679-686.

31. Boudreault J, Desmeules F, Roy J-S, Dionne C, Frémont P, Macdermid JC. The efficacy of oral non-steroidal anti-inflammatory drugs for rotator cuff tendinopathy: A systematic review and meta-analysis. J Rehabil Med 2014;46: 294-306.

32. Jevsevar DS. Treatment of osteoarthritis of the knee: Evidence-based guideline, 2nd edition. J Am Acad Orthop Surg 2013;21:571-576. 